\title{
Influencing Factors Analysis in Simulation of Head-windscreen Laminated Glass Impacting
}

\author{
Na YANG ${ }^{1, a}$, Li-Ren JIANGXIONG ${ }^{1, b}$, Xiao-He TAO ${ }^{1, c}$, Jian-Feng WANG ${ }^{1, d, *}$, \\ Da-Fang WANG ${ }^{1, e}$
}

\author{
${ }^{1}$ School of Automotive Engineering, Harbin Institute of Technology, Weihai 264209, China

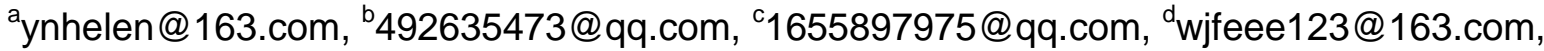 \\ ewdflcjl@163.com
}

${ }^{*}$ Corresponding author

Keywords: Automotive Passive Safety, Laminated Glass, Influencing Factors, Simulation.

\begin{abstract}
In order to reduce head injury in the collision between pedestrian and vehicle, and explore a variety of influence factors of laminated glass on the impact injury. Several laminated glass impact damage effect factors are considered to establish a series of simulation models, crash simulation calculations are conducted using LS-DYNA; In this paper, using finite element model of sedan vehicle, according to EEVC WG17 pedestrian protection legislation and regulations, the finite element model of adult head impactor hitting PVB laminated windscreen was established, and collision simulation analysis was performed using LS -DYNA; secondly, modify related factors of original vehicle laminated glass using HyperMesh, the factors closest to the actual value were obtained. Research results show that ,at the same speed, simply supported constraint form is closer to rubber support constraint form; appropriate size of finite element mesh is $5 \mathrm{~mm}$, and it is closest to the actual value; when the friction coefficient is 0.5 , it is closest to real value. Under the premise of the same speed, with the decrease of elastic modulus, acceleration curve obtained from the simulation analysis deviates more from that obtained from experiment.
\end{abstract}

\section{Introduction}

In all types of accidents, pedestrian mortality accounted for $26 \%$ of total mortality, higher than any other type of traffic accident mortality[1].Therefore, according to the characteristics of domestic road traffic, it is necessary to carried out pedestrian safety studies.

In a car accident, head injury is mainly caused by the collision with the regions of engine hood, PVB laminated windscreen and so on. Windshield is one of the automotive components which causes largest proportion of head impact injury. And the region causing the most serious head impact injury is near the side edges of pillar A and the junction of the windscreen's lower edge with the engine hood.

So the study of windscreen damage under the impact of pedestrian head is important. Due to the affect factors of laminated glass boundary finite element analysis is various, in this paper, the impact affects caused by constraint form of laminated glass, the grid size of the model of the pedestrian's head hitting the windscreen, bulk modulus and contact friction coefficient of PVB material are considered.

\section{Theory about PVB Laminated Windshield Glass}

Polyvinyl butyral, PVB for short, is a condensation product that is generated by reactions of polyvinyl alcohol and n-butyl aldehyde, on acid catalyst. In the research about automotive laminated windshield glass, PVB film is generally regarded as almost incompressible solid.

Figure 1 shows the molecular structure of PVB material [2], it can be seen from the molecular structure that PVB has long branched, and therefore has a longer flexibility, low glass transition temperature, with high tensile strength and impact strength; in addition, since the PVB material has good transparency, good solubility, good light resistance, water resistance, heat resistance, cold 
resistance and film-forming properties, high tensile strength and impact resistance, etc., it has been widely used in the manufacture of laminated safety glass, adhesive, aluminum foil paper, FRP products, and so on.

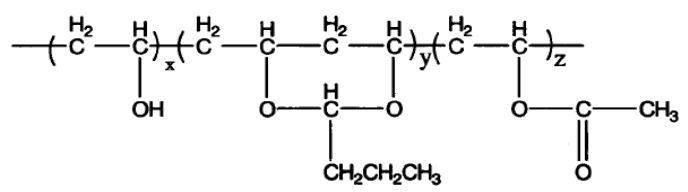

Fig. 1 molecular structure of PVB material

\section{Impact Test of Pedestrian Head Impactor}

In this article, according to EEVC pedestrian safety impactor testing procedures and the relevant technical requirements, establish adult head impactor model; in the process of pedestrian's head Laminated windscreen collision, research the effect of PVB laminated windscreen finite element model parameters on the reliability of the model, and according to the related technical requirements in EEVC pedestrian safety impactor testing procedures, finite element models of adult head impactor and PVB laminated windscreen are established

\section{Pedestrian Safety Test Methods}

The laminated glass used herein, is composed of two layers of glass, the middle layer is made of PVB film, its length and breadth dimensions is $1400 \mathrm{~mm} \times 700 \mathrm{~mm} \times 4.46 \mathrm{~mm}$, the thickness of the upper glass is $2.1 \mathrm{~mm}$, the thickness of the PVB film is $0.76 \mathrm{~mm}$, the thickness of the lower glass is $1.6 \mathrm{~mm}[3]$.

Figure 2 is a theoretical impact model which is about the collision between a head and PVB laminated windshield glass[4]. In this article, simulation analysis of finite element model is performed with the model shown in Figure 2

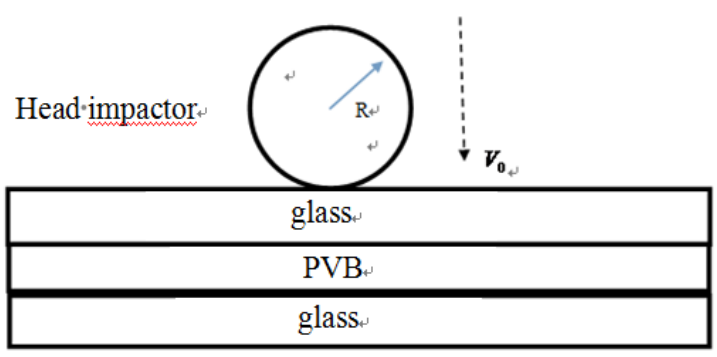

Fig. 2 theoretical impact model

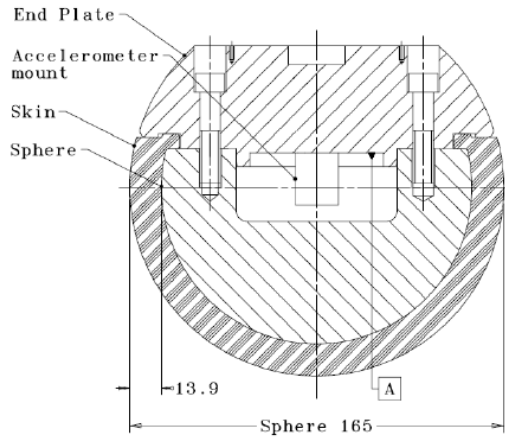

Fig. 3 adult head impactor

\section{Pedestrian Head Impact Tests}

An aluminum sphere is used as the main structure of the adult head impactor in EEVC WG17, the quality of which is $4.8 \pm 0.1 \mathrm{~kg}$. And it is shown in Figure 3. Surface of pedestrian head impactor is wrapped with polyethylene scalp, the entire impactor diameter is $165 \pm 1 \mathrm{~mm}$. According to regulations, it must ensure that the geometric center of the sphere and gravity center of adult head impactor is coincident, or both position errors must not exceed $5 \mathrm{~mm}$. Acceleration sensor is installed in the sphere[5].

The direction of impactor should be parallel to the vertical plane of the test vehicle, and angle error must be controlled within $2^{\circ}$, the collision angle of adult head impactor and the windscreen is measured with the reference of horizontal plane, the angle is $65 \pm 2^{\circ}$, and bump windscreen at speed of $40 \mathrm{~km} / \mathrm{h}$.

In this paper, adult head impactor model is built in CATIA. Adult head impactor consists of the following four parts: upper cover, lower spheres, artificial skin covering the outer surface and 
acceleration sensors. Three-dimensional solid model established by CATAI is imported into HyperMesh to mesh, thereby, the head impact finite element model is established, as is shown in Figure 4.

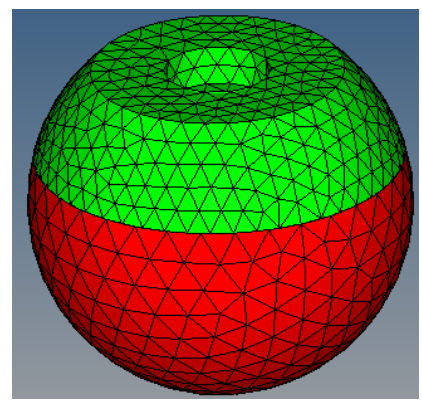

Fig. 4 Adult head impactor finite element model

Tab.1 material parameters of head impactor

\begin{tabular}{|c|c|c|c|c|c|c|}
\hline & \multirow[t]{2}{*}{ Mass $(\mathrm{kg})$} & \multicolumn{2}{|c|}{ Density $\left(\mathrm{kg} \cdot \mathrm{m}^{-3}\right)$} & \multirow{2}{*}{$\begin{array}{c}\text { Scalp bulk } \\
\text { modulus } \\
(\mathrm{GPa})\end{array}$} & \multirow{2}{*}{$\begin{array}{c}\text { Skull elastic } \\
\text { modulus }(\mathrm{GPa})\end{array}$} & \multirow{2}{*}{$\begin{array}{l}\text { Poisson's } \\
\text { ratio }\end{array}$} \\
\hline & & Scalp & Skull & & & \\
\hline Adult & 4.796 & 956 & 2940 & 0.25 & 30 & 0.33 \\
\hline
\end{tabular}

\section{Laminated Windscreen Model}

Now the windshield glass of a sedan vehicle finite element model is extracted as a basic layer glass to establish laminated glass finite element model, the model is shown in Figure 5.

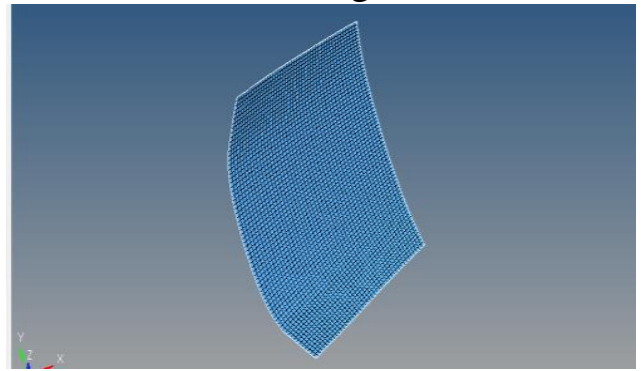

Fig. 5 extracted windshield glass FE model Fig. 6 Windscreen FE model with constraints

Establishment of windscreen finite element model consists of the following steps[7]: establishment of lower glass layer; the establishment of the upper glass layer; the establishment of intermediate PVB film interlayer; establishment of material; establishment of property; identify each component material and property; establishment of contacts ;establishment of binding; establishment of control cards; eventually exported and saved as $\mathrm{K}$ files.

Finite element model is select as shell-body-shell form.Mat123 material is selected on the upper and lower glass layer, mat06 material is selected on PVB film and PVBfacemat09.

\section{Finite Element Analysis of Laminated Glass Affect Factors}

The affect factors of laminated glass for pedestrian protection are various, for example, the contact friction coefficient between the head and the windshield glass, the size of laminated windshield glass finite element grid, constraint forms and so on. This section mainly describes affect analysis of some PVB laminated glass factors which influences pedestrian protection in the pedestrian head impact

\section{Analysis of PVB Laminated Windscreen Constraint Form}

In the simulation analysis, pedestrian protection effectiveness of clamped supported and simply 
supported form of constraint is compared to find out which form is better. In this section, analysis and comparison are mainly conducted by comparing the three sets of data. The only variable is set to the impact velocity, analyze the pedestrian head injured degree at speed of $35 \mathrm{~km} / \mathrm{h}, 40 \mathrm{~km} / \mathrm{h}$.

Fixed Constraint Form. Set the boundary conditions of laminated glass to be four edges fixed support constraint, four edges fixed form of constraints is to confine degrees of freedom in six directions of windshield glass.

Simple Supported Constraint Form. Simulation analysis method and result processing method of simply supported finite element model is the same as those of the fixed constraint finite element model. Figure 10 shows comparison of effective real test simulation results in two constraint forms and at different speeds. (Series 3 is acceleration curve extracted from the test, series 2 is simulation acceleration curve under simply supported constraint, series 1 is simulation acceleration curve under clamped supported constraint).

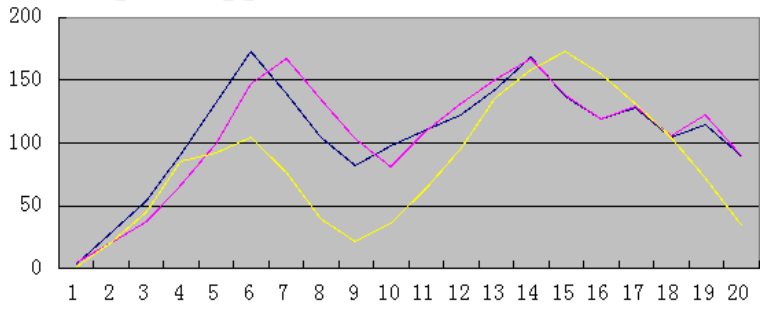

(a) simulation at the speed of $35 \mathrm{~km} / \mathrm{h}$

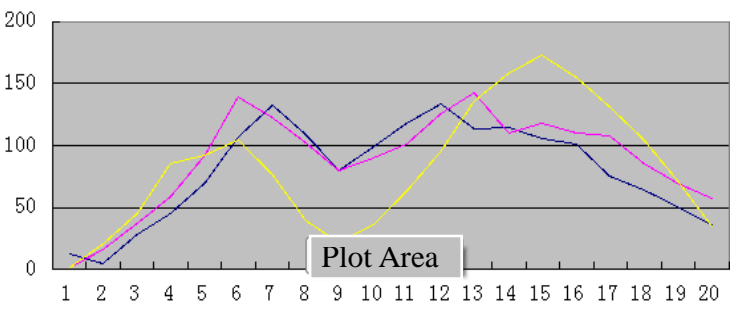

(b)simulation at the speed of $40 \mathrm{~km} / \mathrm{h}$

Fig. 7 Comparison between test simulation and test

By comparative analysis from the above charts, conclusions can be drawn. For PVB laminated glass, the constraint form of windshield used in the test is bond by glass glue. In this section, it can be seen from the comparative experiment between four sides of the fixed support constraint and the simple supported constraint, the simulation acceleration curve of simply supported form of constraint and sticky form constraint is the closest. Therefore, in the separate impact test of head impactor hitting laminated glass, the mechanical characteristics of simply supported constraint can better reflect that of PVB laminated glass.

\section{Effect of Grid Size}

This section focuses on the effect of the density of the grid (size of the grids ) on finite element analysis of laminated glass impact damaged.

In this paper, since the laminated glass studied is seen as a rectangular plate with regular shape, so energy absorption zone mesh shape should be regular. This paper selects grid size $5 \mathrm{~mm}, 7 \mathrm{~mm}$ and $10 \mathrm{~mm}$.For a better comparison, the coefficient of friction of 0.3 and 0.5 were selected to compare and analysis.

Compare the results of the finite element model with different mesh sizes and experimental simulation, plot acceleration curve in the post processing. Figure 8 is about comparative analysis results of different grid sizes simulation.

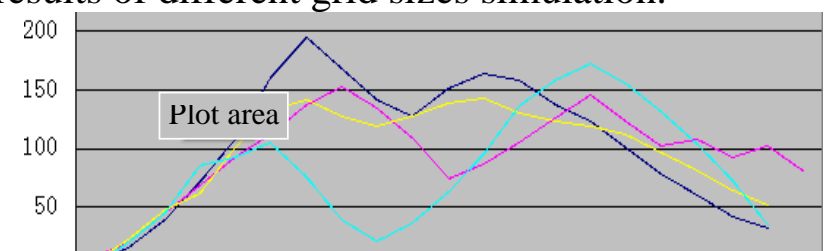

(a)under friction coefficient value of 0.3

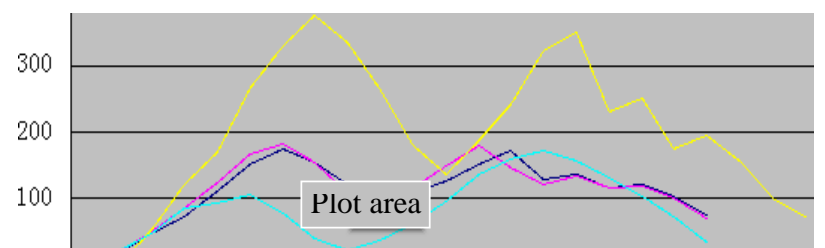

(b) under friction coefficient value of 0.5

Fig. 8 Acceleration curve comparison with different grid size

It can be concluded from comparison and analysis of Figure 8: in the collision experiments of windscreen and the head impactor, under the same friction coefficient, the acceleration curve obtained is closer to the experiment result when the test grid size selects $5 \mathrm{~mm}$. 


\section{Effect of Friction Coefficient}

In this section, contact friction coefficient between the head and the windscreen selects $0.1,0.3,0.5$. To ensure reliability of the results, friction coefficients under different constraints are also compared.

In order to obtain the friction coefficient closest to actual value, in this section, simulation results of finite element model with different friction coefficients are compared with simulation results obtained in the experiment. Figure 9 shows a comparative analysis of experiment simulation results with different constraint forms and different friction coefficients (series 4 is test acceleration curve).

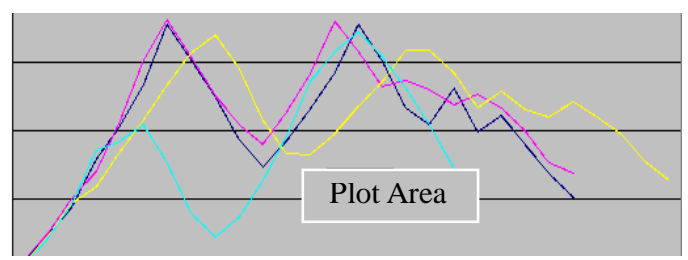

(a) under clamped supported constraint

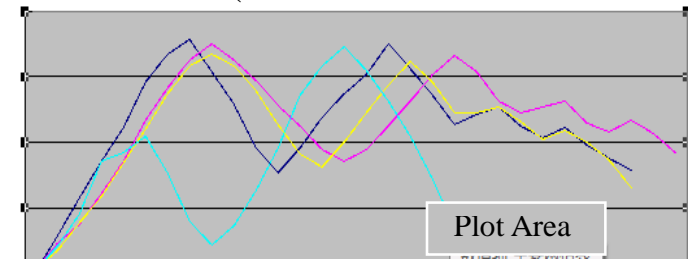

(b) under simply supported constraint

Fig. 9 Acceleration curve comparison with different friction coefficients

It can be concluded from the above figures that, in the pedestrian vehicle collision accident, within a certain range which to meet precision of friction characteristics in various parts on pedestrian and vehicle, when the friction coefficient is 0.3 , acceleration curve obtained is closest to that obtained in the simulation experiment .

\section{Influence of PVB Film Bulk Modulus}

In this paper, bulk modulus values are selected $1.5 \mathrm{GPa}, 2 \mathrm{GPa}, 2.5 \mathrm{GPa}, 3.5 \mathrm{GPa}$, and compare the results at different speeds. Set bulk modulus in BULK option, since the units used in the software is $\mathrm{MPa}$, so unit should be unified as $1500 \mathrm{MPa}, 2000 \mathrm{MPa}, 2500 \mathrm{MPa}, 3000 \mathrm{MPa}$.

Figure 10 shows the comparison between acceleration graph obtained in software simulation at different speeds and different bulk modulus and that obtained in the experiment (Series 5 is the acceleration curve, series 1-4 is respectively simulation acceleration curve with elastic modulus value $1500 \mathrm{MPa}, 2000 \mathrm{MPa}, 2500 \mathrm{MPa}, 3000 \mathrm{MPa})$.
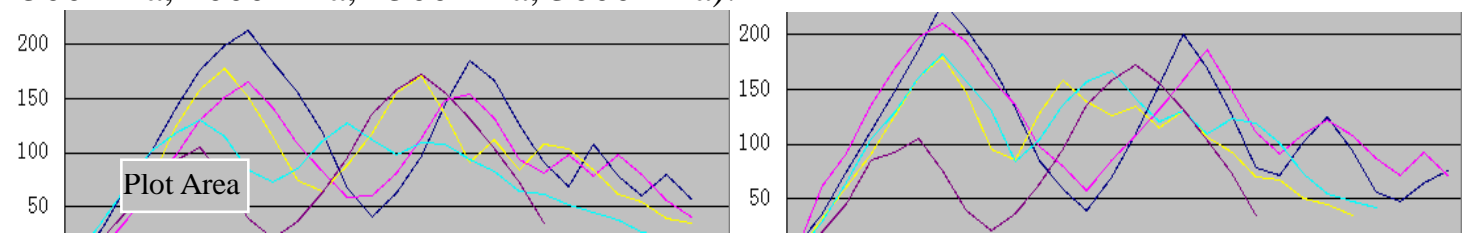

(a) simulation results at speed of $35 \mathrm{~km} / \mathrm{h}$ (b)simulation results at speed of $40 \mathrm{~km} / \mathrm{h}$

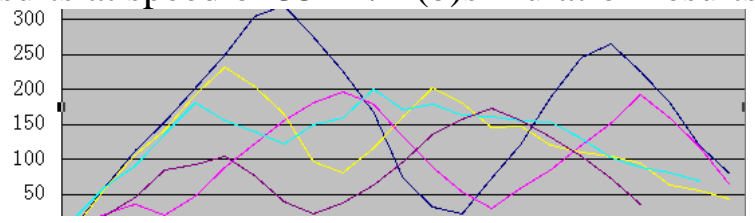

(c)simulation results at speed of $45 \mathrm{~km} / \mathrm{h}$

Fig. 10 Acceleration curve comparison with different bulk modulus

It can be seen from these figures that, in the premise of the same speed, in the process of finite element analysis under different modulus of elasticity, the elastic modulus value adjustments have a greater impact on the energy curve and the acceleration curve. When PVB bulk modulus value is $3000 \mathrm{MPa}$, the result is closest to the experiment.

\section{Conclusions}

(1).In this paper, using finite element model of sedan vehicle, according to EEVC WG17 pedestrian protection legislation and regulations, establish the finite element model of adult head 
impactor hitting PVB laminated windscreen using HyperMesh, and get on a preprocessing; Calculation of the collision process is performed using LS -DYNA; plot the acceleration curve using the post processing software HyperGraph.

(2). The influence of the contact friction coefficient between the head and the windscreen, the size of laminated windscreen FEM finite element grids, PVB film material elastic modulus and constraints form is considered to establish the finite element model and to get on the simulation analysis. And take the comparison on the basic of experiment curve obtained by Qi Liu et al.

In this paper, although to some extent, the different factors are considered to establish finite element model and get on the finite element analysis, but comparative analysis conducted in this article only choose several different parameters, consecutive and consistent is not very good, although several individual parameters can also explain some problems, but the conclusions may not be optimal. We need further improvement and perfection.

\section{References}

[1] Ministry of Public Security Traffic Management Bureau. People's Republic of China road accident statistics compilation ,Masses Press, 1995-2005.

[2] G.D. Li, Y. Zhang. Synthesis and Application of polyvinyl butyral. Dalian: Dalian University of Technology, 2006,15(6).

[3] T.Pyttel ,H. Liebertz ,J. Cai . Failure criterion for laminated glass under impact loading and its application in finite element simulation. International Journal of Impact Engineering, 2011, 38(4).

[4] W. Xu, H.Sun, X.M. Wan. Injury risk analysis of pedestrian head impacting windshield. Automotive Technology, 2010 (1).

[5] J.Xu , Y.B.Li. Crack analysis in PVB laminated windshield impacted by pedestrian head in traffic accident[J]. International Journal of Crashworthiness, 2009, 14(1).

[6] J.Oxley, B.Corben, B.Fildes, et al. Older vulnerable road users-measures to reduce crash and injury risk. Monash University Accident Research Centre Reports, 2004 (218).

[7] L.F. Feng, Z.Y. Xia, Q. He, et al. Micro-buses positive crash analysis based on the LS-DYNA. Journal of Zhengzhou University (Engineering Science), 2013, 34(1). 